\title{
A clinical evaluation of trichomoniasis in San Jose, Costa Rica using the InPouch TV test
}

\author{
K A Borchardt, V Hernandez, S Miller, K Loaiciga, L Cruz, S Naranjo, N Maida
}

\begin{abstract}
Objective-to determine the prevalence of trichomoniasis in San Jose, Costa Rica, comparing two methods, the InPouch ${ }^{\text {TM }}$ TV test and the saline wet mount.

Methods-One hundred symptomatic and asymptomatic female patients at two hospitals and at a sexually transmitted disease (STD) clinic were evaluated. Vaginal discharge was the most prevalent genitourinary abnormality among symptomatic patients. The patients were between 18 and 70 years old. Fifty-seven were from the STD clinic, 43 from the two hospitals. A saline wet mount and a culture were taken from each patient. The culture employed a new procedure for diagnosis of trichomonads, the InPouch ${ }^{\text {TM }}$ TV test (BioMed Diagnostics, San Jose, CA).
\end{abstract}

Results-Thirteen of the 100 patients were culture positive, two of whom were wet mount positive. No wet mount positives were culture negative. Eleven of the positive tests were from the STD clinic and two were from the hospitals.

Conclusions-The results of this initial epidemiologic study indicate a prevalence of $19 \%$ for trichomoniasis in the STD clinic population and $4.6 \%$ in the hospitals group. Trichomonas vaginalis was not diagnosed by laboratory methods prior to this study. The InPouch ${ }^{\text {TM }}$ TV test has a selective fungicidal and bactericidal, enriched proteose-peptone medium which provides a sensitivity of 4 organisms per $\mathrm{ml}$ and a 1 year shelf life at room temperature. This in vitro culture test demonstrated unique capabilities as a transport and culture medium. Its procedure offers simplicity in application and an excellent visualisation of trichomonads.

\section{Introduction}

The flagellated protozoan, Trichomonas vaginalis, is the aetiological agent in one of the most frequently occurring sexually transmitted diseases (STDs). Annually an estimated 180 million cases occur worldwide. ${ }^{1}$ Although trichomoniasis in women may be symptomatic and produce vulvar itching, a purulent discharge, and red punctate areas on the vaginal and cervical mucosa, clinical findings indicate that as many as $50 \%$ of patients are asymptomatic. ${ }^{2}$ Most infected male patients remain asymptomatic. ${ }^{3}$

In both Central and South America, epidemiological data are not available as to the prevalence of trichomoniasis or laboratory methods employed in diagnosis. Presently in Costa Rica, the Papanicolaou-stained gynaecological smear is used as an adjunct to clinical diagnosis.

This study is the first epidemiological investigation of trichomoniasis in San Jose, Costa Rica, evaluating both symptomatic and asymptomatic patients at three clinic sites. Patient specimens were examined by the saline wet mount and in vitro culture. The InPouch ${ }^{\text {TM }} \mathrm{TV}$ test for in vitro culturing of $T$ vaginalis was utilised. This method has previously demonstrated significant advantages over other culture procedures. ${ }^{4}$ This in vitro culture test has a selective fungicidal and bactericidal, enriched proteose-peptone medium which provides a sensitivity of 4 organisms per ml. ${ }^{4}$ The pouch test could not be compared with another in vitro test in this study because of laboratory constraints.

Materials and methods

The three clinic sites involved in this study were Hospital San Juan de Dios, Clinica Biblica, and Lucha Antivenerea de Costa Rica. One hundred symptomatic and asymptomatic patients were sampled for trichomoniasis. The patients' hospital number, age, and symptoms were recorded.

All wet mounts were performed by one of the authors (KAB) who is an experienced microscopist in protozoan infections. Sterile cotton-tipped swabs were used to obtain specimens from the posterior fornix of the vagina. Each swab was placed in a sterile glass tube containing $0.5 \mathrm{ml}$ of sterile saline immediately after sampling. The specimen was eluted from the cotton swab by vigorously twirling the stem of the swab. The saline specimen was held at $27^{\circ} \mathrm{C}$ and evaluated within 60 minutes. A drop of the specimen was placed on a glass slide, cover slipped, and examined microscopically under low power $(\times 100)$. Then the swab was placed in the saline mixture and used to

Table 1 Patients tested for trichomoniasis in San fose, Costa Rica

\begin{tabular}{llc}
\hline & \multicolumn{2}{l}{ Numbers of Patients } \\
\cline { 2 - 3 } Clinical Location & Symptomatic & Asymptomatic \\
\hline Lucha Antivenerea de Costa Rica & 38 & 19 \\
Hospital San Juan de Dios & 17 & 9 \\
Clinica Biblica & 14 & 3 \\
Total & 69 & 31 \\
\hline
\end{tabular}


Table 2 Patients positive for Trichomonas vaginalis

\begin{tabular}{rllll}
\hline Patient & Age (years) & Symptoms & Wet mount & Time culture positive \\
\hline 1 & 28 & Asymptomatic & Positive & 24 hours \\
2 & 32 & Asymptomatic & Positive & 24 hours \\
3 & 26 & Vaginal discharge & Negative & 24 hours \\
4 & 26 & Vaginal discharge & Negative & 24 hours \\
5 & 32 & Vaginal discharge & Negative & 24 hours \\
6 & 33 & Asymptomatic & Negative & 24 hours \\
7 & 33 & Dyspareunia & & 24 hours \\
& & Vaginal discharge & Negative & 48 hours \\
8 & 55 & Vagingal discharge & Negative & 5 days \\
9 & 47 & Itching & Negative & 5 days \\
10 & 22 & Vaginal discharge & Negative & 5 days \\
11 & 23 & Yeast & Negative & 5 days \\
12 & 45 & Vaginal discharge & Negative & \\
13 & 50 & Itching & Unknown symptoms &
\end{tabular}

Except for patient No 11 from Hospital San Juan de Dios and patient No 13 from Clinica Biblica, the others were from Lucha Antivenerea de Costa Rica.

inoculate the InPouch ${ }^{\mathrm{TM}} \mathrm{TV}$ as previously described. ${ }^{4}$ Tests were incubated at $37^{\circ} \mathrm{C}$, examined at $24 \mathrm{~h}$ and when negative at $48 \mathrm{~h}$ and 5 days.

\section{Results}

In this study, 100 symptomatic and asymptomatic female patients between the ages of 18 and 70 years were studied for trichomoniasis by saline wet mount and culture with the InPouch $^{\mathrm{TM}}$ TV test. Table 1 presents the number of patients examined at each site and their clinical status. The majority of the patients, 57, were examined at the Lucha Antivenerea Costa Rica (the STD clinic). Twenty six patients were examined at the Hospital San Juan de Dios, and 17 at the Clinica Biblica. Four of the patients tested at Hospital San Juan de Dios and two at Clinica Biblica were diagnosed as positive for trichomoniasis by Papanicolaou smears. None of these six patients were positive either by saline wet mount or in vitro culture.

In Table 2 , the patients positive for $T$ vaginalis are listed as to their age, symptoms, which tests were positive, and the incubation period for a positive culture. Their ages ranged from 23 to 55 years, and three were asymptomatic. Two of the patients were both saline wet mount and culture positive. Eight of the cultures were positive within $24 \mathrm{~h}, 1$ at $48 \mathrm{~h}$, and 4 at 5 days.

\section{Discussion}

In evaluating a specimen for trichomonads by saline wet mount, the sensitivity of the test varies by the number of trichomonads present, the length of time the specimen has remained at room temperature, and the experience of the microscopist. ${ }^{5}$ The results of this study corroborate the importance of an in vitro culture for accurate diagnosis because only two of 13 patients were saline wet mount positive.

The InPouch ${ }^{\text {TM }}$ TV test was used in this epidemiological evaluation in a tropical country because of the test's unique capabilities as a transport and culture medium, simplicity in application, and in excellent visualisation of trichomonads. Its sensitivity is $\mathbf{4}$ organisms for positive growth. ${ }^{4}$ Because of the antibacterial and antifungal agents in the medium, the test's specificity is significant even in patients with polymicrobic infections.

The InPouch ${ }^{\mathrm{TM}}$ TV test can be used for rapid (18 h) diagnosis. A viewer is employed to immobilise the culture medium and allows the pouch to be evaluated within 2 minutes. ${ }^{4}$ The test is self-contained and requires nothing other than a microscope for viewing. Two other important advantages are that the medium has a 1 year shelf life at room temperature and the test can serve as a transport medium for several days while maintaining the viability of the trichomonads.

Six patients were diagnosed positive for trichomoniasis by cytological studies. None of these patients were either saline wet mount or culture positive. This confirms the findings of Thomason et al ${ }^{6}$ who reported that the Papanicolaou smear was the least specific and sensitive method of detecting $T$ vaginalis. Negative saline wet mount tests for trichomonads in women demonstrate the lowest sensitivity and lowest positive predictive values and therefore may result in an incorrect diagnosis. ${ }^{5}$ Patients either in a low predictive setting or with negative wet mounts should have confirmation tests. $^{5}$

Misdiagnosis of trichomoniasis is particularly important in patients who have had abdominal hysterectomies. Trichomoniasis vaginitis and bacterial vaginosis are risk factors for cuff cellulitis. ${ }^{7}$

An observation that positive trichomonad cultures were found in four patients who were $45,47,50$, and 55 years of age indicates the possible prevalence of this infection in patients of that age. Two of these were diagnosed from out-patient clinics and the other were from the STD clinic. It could be important to quantitate their period of infection and potential nidus of infection. These represent unrecognised cases that could increase the incidence rate in the community.

The results of this study demonstrate that trichomoniasis presents a significant STD problem in Costa Rica in one group of patients examined at the STD clinic. The $19 \cdot 2 \%$ of 57 patients who were positive represents an important reservoir of trichomoniasis in this population. It is important to emphasise that of the 100 patients tested in this study, the other 43 examined at the 2 hospitals were not all symptomatic for vaginal infections. 
1 Krieger JN. Urologic aspects of trichomoniasis. Investigative Urology 1981;18:411-7.

2 Bickley LS, Krisher KK, Punsalang A.Jr, Trupei MA Reichman RC, Menegus MA. Comparison of direct fluorescent antibody, acridine orange, wet mount, and culture for detection of Trichomonas vaginalis in women attending a public sexually transmitted diseases clinic. attending a public sexually transmitted disease
Sexually Transmitted Diseases 1989;16:127-31.

3 Soultz JW, Toffler WL. Trichomonas infection in men American Family Physician 1989;39:177-80.

4 Borchardt KA, Smith RF. An evaluation of an InPouch ${ }^{\mathrm{TM}}$ TV culture method for diagnosing Trichomonas vaginalis infection. Genitourin Med 1991;67:149-52.
5 Krieger JN, Tam MR, Stevens CE, et al. Comparison of conventional wet-mount examination with cytologic studies, cultures, and monoclonal antibody staining of direct specimens. FAMA 1988;259:1223-7.

6 Thomanson JL, Gelbart SM, Sobun JF, Schulien MB, Hamilton PR. Comparison of four methods to detect Trichomonas vaginalis. f Clin Microbiol 1988;26: 1869-70.

7 Soper DE, Bump RC, Hurt WG. Bacterial vaginosis and trichomoniasis vaginitis are risk factors for cuff cellulitis after abdominal hysterectomy. Am $\mathcal{F}$ of Obstet Gynecol 1990;163:1016-21. 